\title{
Stationary untrapped boundary conditions in general relativity
}

\author{
Roh-Suan Tung \\ Center for Astrophysics, Shanghai Normal University, Shanghai 200234, China \\ E-mail: tung@shnu.edu.cn
}

\begin{abstract}
A class of boundary conditions for canonical general relativity are proposed and studied at the quasi-local level. It is shown that for untrapped or marginal surfaces, fixing the area element on the 2-surface (rather than the induced 2-metric) and the angular momentum surface density is enough to have a functionally differentiable Hamiltonian, thus providing definition of conserved quantities for the quasi-local regions. If on the boundary the evolution vector normal to the 2 -surface is chosen to be proportional to the dual expansion vector, we obtain a generalization of the Hawking energy associated with a generalized Kodama vector. This vector plays the role for the stationary untrapped boundary conditions which the stationary Killing vector plays for stationary black holes. When the dual expansion vector is null, the boundary conditions reduce to the ones given by the non-expanding horizons and the null trapping horizons.
\end{abstract}

\section{Introduction}

Traditional description of black holes in terms of event horizons is inadequate for many physical applications, especially in the cases of non-stationary spacetimes. Quasi-local notions of trapped and marginal surfaces have now been found to be more useful for these cases within the framework of trapping, isolated, and dynamical horizons [1, 2, 3, 4, 5, 6, 7, 8, 9, 10, 11]. These frameworks enable one to significantly extend the laws of black hole mechanics to the dynamical regime with the associated notions of energy, angular momentum and their fluxes, and have been applied to several problems in mathematical general relativity, numerical relativity, and quantum gravity [12]. These progresses on black hole dynamics lead to a question whether we can generalize the conservation laws for isolated and dynamical trapping horizons to general untrapped regions so that we can study the change of energy, angular momentum and their fluxes for untrapped strong gravitating systems, e.g., before the black hole horizon was formed.

The question of how to define energy and angular momentum for untrapped surfaces has been raised for a while in searching for the "quasi-local energy-momentum and angular momentum" [13]. The goal has been to find a suitable definition of total energymomentum and angular-momentum, surrounded by a spacelike 2 -surface $S$, with $\mathbb{S}^{2}$ topology, in 4-dimensional spacetime $M$. The construction is quasi-local in the sense 
that it refers only to the geometry of $S$ (intrinsic metric, first fundamental form), the extrinsic curvatures (second fundamental forms) and the connection 1-forms on the normal bundle (normal fundamental forms) for its embedding in $M$.

A systematic way to study conserved quantities is through the Hamiltonian. For the existence of a functionally differentiable Hamiltonian for General Relativity, it is necessary to impose suitable boundary conditions. The allowed boundary conditions for finite spatial 2-surfaces were studied previously with Dirichlet and Neumann boundary conditions [14, 15]. This extends the requirement of the functional differentiability of the Hamiltonian, considered first by Regge and Teitelboim [16] for spatial infinity to the finite spatial 2-surfaces. Especially interesting boundary conditions are the Dirichlet boundary conditions which fix the induced metric on the 2-surfaces.

The conditions were recently relaxed by Szabados who showed that instead of fixing the full induced metric on the boundary, fixing the area element is enough. Together with conditions that the lapse is vanishing on and the shift is tangent to the boundary and is divergence free, he showed that the Hamiltonian is functionally differentiable, and that in the large sphere limit the conserved quantities derived from the Hamiltonian behave as the spatial components of the total angular momentum [17]. This leads to a question whether or not the condition of vanishing lapse can be relaxed, and what additional conditions should be imposed so that a functionally differentiable Hamiltonian can provide a definition of energy-momentum for the quasi-local region.

In this paper, we propose a class of quasi-local boundary conditions for canonical general relativity. It is shown that for untrapped or marginal surfaces, fixing the area element on the 2-surface $S$ (rather than the induced 2-metric) is enough to have a functionally differentiable Hamiltonian, thus providing definition of conserved quantities for the quasi-local regions that allows the geometry outside to be dynamical and admit gravitational and other radiation. For gravitating systems including angular momentum, we further fix the "angular momentum surface density" at boundary to obtain a generalized definition of quasi-local energy including angular momentum. These boundary conditions characterize the equilibrium situation for regions bounded by untrapped or marginal surfaces.

The evolution vector can be chosen freely. If on the boundary the evolution vector normal to the 2-surface is chosen to be proportional to the dual expansion vector, we obtain a generalization of the Hawking energy associated with a generalized Kodama vector. This vector plays the role for these stationary untrapped boundary conditions which the stationary Killing vector plays for stationary black holes. When the dual expansion vector is null, the boundary conditions reduce to the ones given by null trapping horizons and non-expanding horizons.

We begin with the geometry of an untrapped 2-surface $S$ embedded in a 4 dimensional spacetime $M$. Introduce a set of orthonormal vectors $e_{0}, e_{1}, e_{2}, e_{3}$ adapted to the 2-surface $S$, with $e_{0}$ and $e_{1}$ being the set of timelike and spacelike unit normals to $S$ and $e_{A}=\left[e_{2}, e_{3}\right]$ being tangent to $S$. The extrinsic curvatures of $S$ with respect to $e_{0}$ and $e_{1}$ directions are given by $k\left(e_{0}\right)_{A B}=g\left(e_{B}, \nabla_{A} e_{0}\right)=-\Gamma_{0 B A}$ and 
$k\left(e_{1}\right)_{A B}=g\left(e_{B}, \nabla_{A} e_{1}\right)=-\Gamma_{1 B A}$. The connection 1-forms in the normal bundle are given by $\varpi_{A}=g\left(e_{1}, \nabla_{A} e_{0}\right)=-\Gamma_{01 A}$. Here $\Gamma_{I J K}=-g\left(e_{J}, \nabla_{K} e_{I}\right)$ are Ricci rotation coefficients. Under a boost transformation of $e_{0}$ and $e_{1}$,

$$
\begin{aligned}
& e^{\prime}{ }_{0}=e_{0} \cosh u+e_{1} \sinh u, \\
& e^{\prime}{ }_{1}=e_{0} \sinh u+e_{1} \cosh u,
\end{aligned}
$$

they transform as

$$
\begin{aligned}
& k\left(e_{0}^{\prime}\right)_{A B}=k\left(e_{0}\right)_{A B} \cosh u+k\left(e_{1}\right)_{A B} \sinh u, \\
& k\left(e_{1}^{\prime}\right)_{A B}=k\left(e_{0}\right)_{A B} \sinh u+k\left(e_{1}\right)_{A B} \cosh u, \\
& \varpi^{\prime}{ }_{A}=\varpi_{A}-\nabla_{A} u .
\end{aligned}
$$

The expansion vector $H$, and the dual expansion vector $H_{\perp}$ are defined with the trace of the extrinsic curvatures $k\left(e_{0}\right)$ and $k\left(e_{1}\right)$,

$$
\begin{aligned}
& H=k\left(e_{1}\right) e_{1}-k\left(e_{0}\right) e_{0}, \\
& H_{\perp}=k\left(e_{1}\right) e_{0}-k\left(e_{0}\right) e_{1},
\end{aligned}
$$

where $H$ and $H_{\perp}$ are also known as the mean curvature vector and the dual mean curvature vector respectively. These vectors are independent of choice of normal frames

for the 2-surface. They are invariant under the boost transformation (11). Thus, they depend only on the 2-surface $S$ and constitute a set of natural normal vectors for $S$ [18].

A 2-surface $S$ is trapped if $k\left(e_{1}\right)^{2}>k\left(e_{0}\right)^{2}$, untrapped if $k\left(e_{1}\right)^{2}<k\left(e_{0}\right)^{2}$, and marginal if $k\left(e_{1}\right)^{2}=k\left(e_{0}\right)^{2}$, everywhere on $S$. Untrapped surfaces are also called mean convex surfaces. The dual expansion vector $H_{\perp}$ is always timelike for untrapped surfaces, null for marginal surfaces [18, 14, 15]. Note that, on $S$, the trace of the extrinsic curvature is zero along the direction of the dual expansion vector, i.e. $\left.k\left(H_{\perp}\right)\right|_{S}=0$, thus we have

$$
\left.£_{H_{\perp}} \varrho\right|_{S}=0,
$$

where $\varrho$ is the area element of $S$.

\section{Conserved quantities derived from a hamiltonian}

For a general diffeomorphism-invariant field theory in four dimensions with a Lagrangian 4-form $\mathcal{L}(\phi)$, where $\phi$ denotes an arbitrary collection of dynamical fields, the field equations, $\mathcal{E}=0$, are obtained by computing the first variation of the Lagrangian,

$$
\delta \mathcal{L}=d \Theta(\phi, \delta \phi)+\mathcal{E} \delta \phi,
$$

where $\Theta(\phi, \delta \phi)$ is the symplectic potential 3-form. For any diffeomorphism generated by a smooth vector field $\xi$,

$$
d i_{\xi} \mathcal{L}=£_{\xi} \mathcal{L}=d \Theta\left(\phi, £_{\xi} \phi\right)+\mathcal{E} £_{\xi} \phi,
$$

where $£_{\xi}$ denotes the Lie derivative and $i_{\xi}$ is the inner product, one can define a conserved Noether current 3-form $J(\xi)$ by

$$
J(\xi)=\Theta\left(\phi, £_{\xi} \phi\right)-i_{\xi} \mathcal{L}(\phi),
$$


such that the Noether current $J(\xi)$ is closed $\left(d J(\xi)=-\mathcal{E} £_{\xi} \phi \simeq 0\right)$ when the field equations are satisfied. Locally there exist a 2 -form $Q(\xi)$ (called the Noether charge) such that $J(\xi)=d Q(\xi)$. The variation of the Noether current 3-form is given by

$$
\delta J(\xi)=\omega\left(\phi, \delta \phi, £_{\xi} \phi\right)+d\left(i_{\xi} \Theta(\phi, \delta \phi)\right),
$$

where $\omega$ is the symplectic current 3 -form defined by

$$
\omega\left(\phi, \delta_{1} \phi, \delta_{2} \phi\right)=\delta_{1} \Theta\left(\phi, \delta_{2} \phi\right)-\delta_{2} \Theta\left(\phi, \delta_{1} \phi\right)
$$

Its integral over a 3 -surface $\Sigma$ defines the presymplectic form $\Omega$. If the presymplectic form is a total variation

$$
\Omega\left(\phi, \delta \phi, £_{\xi} \phi\right) \equiv \int_{\Sigma} \omega\left(\phi, \delta \phi, £_{\xi} \phi\right)=\delta \mathbb{H}(\xi),
$$

for some function $\mathbb{H}(\xi)$ on the field space, then $\mathbb{H}(\xi)$ is conserved along $\xi$, i.e. we have $£_{\xi} \mathbb{H}(\xi)=0$. The function $\mathbb{H}(\xi)$ is called the Hamiltonian conjugate to $\xi$ $[19,20,21,22,23]$. Note that on shell, the presymplectic form is given by

$$
\int_{\Sigma} \omega\left(\phi, \delta \phi, £_{\xi} \phi\right)=\int_{\Sigma} \delta J(\xi)-d\left(i_{\xi} \Theta\right)=\oint_{S} \delta Q(\xi)-i_{\xi} \Theta .
$$

For General Relativity we begin with the Hilbert action,

$$
S=\int \mathcal{L}=\int R^{a b} \wedge *\left(\vartheta_{a} \wedge \vartheta_{b}\right)
$$

where $R^{a b}=d \Gamma^{a b}+\Gamma^{a}{ }_{c} \wedge \Gamma^{c b}$ is the curvature 2-form constructed by the connection 1-form $\Gamma^{a b}, *\left(\vartheta^{a} \wedge \vartheta^{b}\right)=\frac{1}{2} \epsilon^{a b}{ }_{c d} \vartheta^{c} \wedge \vartheta^{d}$, and $g=\eta_{a b} \vartheta^{a} \otimes_{s} \vartheta^{b}$ is the metric, where $\eta_{a b}=\operatorname{diag}(-1,1,1,1)$ and $\vartheta^{a}$ is the orthonormal frame 1 -form field. A variation of the Lagrangian gives,

$$
\begin{aligned}
\delta \mathcal{L}= & R^{a b} \wedge \epsilon_{a b c d} \vartheta^{c} \wedge \delta \vartheta^{d}+\delta \Gamma^{a b} \wedge D *\left(\vartheta_{a} \wedge \vartheta_{b}\right) \\
& +d\left(\delta \Gamma^{a b} \wedge *\left(\vartheta_{a} \wedge \vartheta_{b}\right)\right)
\end{aligned}
$$

which identifies the symplectic potential $\Theta(\phi, \delta \phi)=\delta \Gamma^{a b} \wedge *\left(\vartheta_{a} \wedge \vartheta_{b}\right)$. The Noether current 3 -form is given by

$$
J(\xi)=d Q(\xi)=d\left[\left(i_{\xi} \Gamma^{a b}\right) *\left(\vartheta_{a} \wedge \vartheta_{b}\right)\right],
$$

where $Q(\xi)$ is the Noether charge 2-form and we assume the field equations are satisfied. The presymplectic form is given by

$$
\int_{\Sigma} \omega=\oint_{S} \delta Q(\xi)-i_{\xi} \Theta=\oint_{S} C_{1}(\xi)+\oint_{S} C_{2}(\xi),
$$

where, for convenience, we define

$$
C_{1}(\xi)=\frac{1}{2} i_{\xi} \Gamma^{a b} \delta\left(\epsilon_{a b c d} \vartheta^{c} \wedge \vartheta^{d}\right),
$$

and

$$
C_{2}(\xi)=i_{\xi} \vartheta^{c} \wedge \delta \Gamma^{a b} \wedge \epsilon_{a b c d} \vartheta^{d} .
$$


Let us first expand $C_{1}$ to the normal and tangent components,

$$
\begin{aligned}
\oint_{S} C_{1}(\xi)= & \oint_{S} i_{\xi} \Gamma^{01} \delta\left(\epsilon_{01 A B} \vartheta^{A} \wedge \vartheta^{B}\right)+\oint_{S} i_{\xi} \Gamma^{A B} \delta\left(\epsilon_{A B 01} \vartheta^{0} \wedge \vartheta^{1}\right) \\
& +2 \oint_{S} i_{\xi} \Gamma^{0 A} \delta\left(\epsilon_{0 A 1 B} \vartheta^{1} \wedge \vartheta^{B}\right)+2 \oint_{S} i_{\xi} \Gamma^{1 A} \delta\left(\epsilon_{1 A 0 B} \vartheta^{0} \wedge \vartheta^{B}\right) .(19
\end{aligned}
$$

Let $P_{S}$ be the projection onto $S$, we have $P_{S} \vartheta^{0}=P_{S} \vartheta^{1}=0$. Moreover, assuming $\delta\left(P_{S}\right)=0$, we have

$$
P_{S} \delta \vartheta^{0}=\delta\left(P_{S} \vartheta^{0}\right)-\left(\delta P_{S}\right) \vartheta^{0}=0
$$

and likewise $P_{S} \delta \vartheta^{1}=\delta\left(P_{S} \vartheta^{1}\right)-\left(\delta P_{S}\right) \vartheta^{1}=0$. Thus the term with $C_{1}$ reduces to

$$
\oint_{S} C_{1}(\xi)=2 \oint_{S} i_{\xi} \Gamma^{01} \delta \varrho
$$

where $\varrho=\frac{1}{2}\left(\epsilon_{01 A B} \vartheta^{A} \wedge \vartheta^{B}\right)$ is the area element on $S$.

Similarly for $C_{2}$, by a projection onto the 2-surface $S$, it is straightforward to show that the following identity holds on $S$ for any vector field $V=V^{a} e_{a}$ :

$$
\left.V^{c} \delta \Gamma^{a b} \wedge \epsilon_{a b c d} \vartheta^{d}\right|_{S}=-\left.2 \varrho\left(V^{0} \delta k\left(e_{1}\right)+V^{1} \delta k\left(e_{0}\right)-V^{A} \delta \varpi_{A}\right)\right|_{S}
$$

Using this identity, we obtain

$$
\oint_{S} C_{2}(\xi)=-\oint_{S} 2 \varrho\left(i_{\xi} \vartheta^{0} \delta k\left(e_{1}\right)+i_{\xi} \vartheta^{1} \delta k\left(e_{0}\right)-i_{\xi} \vartheta^{A} \delta \varpi_{A}\right) .
$$

By (21) and (23), the full symplectic form is,

$$
\begin{aligned}
\int_{\Sigma} \omega & =\oint_{S} C_{1}(\xi)+C_{2}(\xi) \\
& =\oint_{S} 2 i_{\xi} \Gamma^{01} \delta \varrho-\oint_{S} 2 \varrho\left(i_{\xi} \vartheta^{0} \delta k\left(e_{1}\right)+i_{\xi} \vartheta^{1} \delta k\left(e_{0}\right)-i_{\xi} \vartheta^{A} \delta \varpi_{A}\right) .
\end{aligned}
$$

This is the key equation for our discussion.

\section{Energy}

We assume that the timelike (or null) vector $\xi$ is fixed on $S$,

$$
\left.\delta \xi\right|_{S}=0, \quad \text { (Boundary Condition I), }
$$

moreover, we assume

$$
\left.\delta \varrho\right|_{S}=0, \quad \text { (Boundary Condition II), }
$$

i.e. the area element of the 2 -surface $\varrho$ is fixed.

We first consider a special case by assuming that, on $S,\left.i_{\xi} \vartheta^{A}\right|_{S}=0$ (this condition will be relaxed in the next section). Using the boundary conditions (I, II),

$$
\oint_{S} C_{2}(\xi)=-\delta \oint_{S} \varrho i_{\xi}\left(\vartheta^{0} k\left(e_{1}\right)+\vartheta^{1} k\left(e_{0}\right)\right)
$$

is a total variation, and

$$
\oint_{S} C_{1}(\xi)=2 \oint_{S} i_{\xi} \Gamma^{01} \delta \varrho=0
$$


Thus with boundary conditions (I) and (II), the full symplectic form,

$$
\int_{\Sigma} \omega=-\delta \oint_{S} \varrho i_{\xi}\left(\vartheta^{0} k\left(e_{1}\right)+\vartheta^{1} k\left(e_{0}\right)\right)=\delta \mathbb{E}_{H}(\xi)
$$

is a total variation. The Hamiltonian $\mathbb{E}_{H}(\xi)$ associated with the vector $\xi$ is given by

$$
\mathbb{E}_{H}(\xi)=\oint_{S}\left(f(\varrho)-i_{\xi}\left(\vartheta^{0} k\left(e_{1}\right)+\vartheta^{1} k\left(e_{0}\right)\right)\right) \varrho .
$$

where $f(\varrho)$ is a function of the area element. The evolution vector $\xi$ can be chosen freely. By choosing $\xi$ to be timelike or spacelike vector, the Hamiltonian gives energy or momentum respectively. Here we assume $\xi$ is timelike.

Note that because of equation (5), the boundary conditions (I) and (II) are both satisfied if we replace the variation with the Lie derivative with respect to $\xi\left(\delta=£_{\xi}\right)$, and assume that on $S$, the evolution vector $\xi$ is given by

$$
\left.\xi\right|_{S}=h(\varrho) H_{\perp},
$$

where $H_{\perp}$ is the dual expansion vector and $h(\varrho)$ is a function of the area element on the (untrapped or marginal) 2-surface $S$.

The boundary condition II implies that the area is conserved along the dual expansion vector. Since the area of each cross section does not change along the dual mean curvature vector direction, we called it the stationary untrapped boundary conditions.

Note that because of equation (131),$\left.\xi\right|_{S}=h(\varrho) H_{\perp}$, so by equation (4), we have, on $S, i_{\xi} \vartheta^{0}=h(\varrho) k\left(e_{1}\right), i_{\xi} \vartheta^{1}=-h(\varrho) k\left(e_{0}\right)$, and $i_{\xi} \vartheta^{A}=0$. Thus the Hamiltonian $\mathbb{E}_{H}(\xi)$ associated with the vector $\xi$ is given by

$$
\mathbb{E}_{H}(\xi)=\oint_{S}\left(f(\varrho)-h(\varrho) H^{2}\right) \varrho .
$$

The free functions of the area element, $f(\varrho)$ and $h(\varrho)$, can be chosen such that the expression gives ADM mass at spatial infinity and irreducible mass at marginal surfaces $H=0$. This can be done by letting $f(\varrho)$ to be $1 /(8 \pi \mathbb{R})$ and let $h(\varrho)$ to be $\mathbb{R} /(32 \pi)$, where $\mathbb{R}$ is the area radius given by

$$
\mathbb{R}=\sqrt{\frac{1}{4 \pi} \oint_{S} \varrho .}
$$

This leads to the energy expression,

$$
\mathbb{E}_{H}(\xi)=\frac{\mathbb{R}}{2}\left(1-\frac{1}{16 \pi} \oint_{S} H^{2} \varrho\right),
$$

which is precisely the Hawking energy [24] .

\section{Angular momentum}

The equation (24) can also be used to define angular momentum. Let $\left.\xi\right|_{S}=(8 \pi)^{-1} \psi$ be a vector tangent to $S$ satisfying $\left.i_{\psi} \vartheta^{0}\right|_{S}=0$ and $\left.i_{\psi} \vartheta^{1}\right|_{S}=0$, and

$$
\left.\delta \psi\right|_{S}=0, \quad\left(\text { Boundary Condition } \mathrm{I}^{\prime}\right),
$$


and

$$
\left.\varpi_{A} \delta \vartheta^{A}\right|_{S}=0, \quad \text { (Boundary Condition A), }
$$

then

$$
\int_{\Sigma} \omega=\frac{1}{8 \pi} \delta \oint_{S} \psi^{A} \varpi_{A} \varrho=\delta \mathbb{J}(\psi)
$$

is a total variation, where $\psi^{A}=i_{\psi} \vartheta^{A}$. The Hamiltonian associated with $\psi$ is the angular momentum given by

$$
\mathbb{J}(\psi)=\frac{1}{8 \pi} \oint_{S} j \varrho=\frac{1}{8 \pi} \oint_{S} \psi^{A} \varpi_{A} \varrho,
$$

with the "angular momentum surface density" given by $j=\psi^{A} \varpi_{A}$.

Because of the gauge freedom in choosing the normal fundamental forms $\varpi_{A}$ (equation (2) ), the definition of angular momentum is not unique. A further condition which makes it unique is

$$
\left.\delta_{\psi} \varrho\right|_{S}=\left.£_{\psi} \varrho\right|_{S}=0, \quad \text { (Boundary Condition } \mathrm{II}^{\prime} \text { ). }
$$

Here $\psi$ generates a symmetry of the area form rather than the whole metric. This implies that $\psi$ has vanishing transverse divergence $\nabla_{A} \psi^{A}=0$. Under the gauge freedom for the normal fundamental forms $\varpi_{A} \mapsto \varpi_{A}-\nabla_{A} u$, the angular momentum formula

$$
\oint_{S} \psi^{A} \varpi_{A} \mapsto \oint_{S} \psi^{A}\left(\varpi_{A}-\nabla_{A} u\right)=\oint_{S} \psi^{A} \varpi_{A}-\nabla_{A}\left(\psi^{A} u\right)=\oint_{S} \psi^{A} \varpi_{A},
$$

is invariant. Thus the angular momentum formula is uniquely defined on $S$ (up to the choice of $\psi^{A}$ ). This condition is also given in [6, 10, 9, 11] for dynamical trapping horizons and was also used by Szabados [17] as a Hamiltonian boundary condition for quasi-local angular momentum.

We can now extend the stationary untrapped boundary conditions to include angular momentum. Similar to the discussion in the previous section, we require that $\left.\delta \xi\right|_{S}=0$ (Boundary Condition I) and $\left.\delta \varrho\right|_{S}=0$ (Boundary Condition II) are satisfied. In addition, we further require that Boundary Condition $\mathrm{A}$ is satisfied. This implies that the angular momentum surface density $j$ is fixed on $S$, i.e.

$$
\left.\delta j\right|_{S}=0, \quad \text { (Boundary Condition III). }
$$

The full symplectic form is then given by applying the boundary conditions to the equation (24),

$$
\int_{\Sigma} \omega\left(\phi, \delta \phi, £_{\xi} \phi\right)=\delta \mathbb{E}(\xi)
$$

where the Hamiltonian associated with $\xi$ is now given by

$$
\mathbb{E}(\xi)=\oint_{S}\left(f(\varrho, j)-i_{\xi}\left(\vartheta^{0} k\left(e_{1}\right)+\vartheta^{1} k\left(e_{0}\right)\right)\right) \varrho .
$$

A natural choice of the evolution vector on $S$ is

$$
\left.\xi\right|_{S}=h(\varrho, j) H_{\perp}-\Omega(\varrho, j) \psi,
$$


which is assumed to be timelike or null. The free functions $h(\varrho, j)$ and $\Omega(\varrho, j)$ (angular speed) are now functions of the area element $\varrho$ and the angular momentum surface density $j$.

Now we replace the variation $\delta$ by the Lie derivative $£_{\xi}$, the boundary condition (I) is automatically satisfied $\left(£_{\xi} \xi=0\right)$. By equation (44), the boundary condition (II) is satisfied with the following condition on $S$ :

$$
\left.£_{\psi} \varrho\right|_{S}=0 .
$$

The boundary condition (III) is satisfied if the vector field $\psi$ is transported by $H_{\perp}$,

$$
\left.£_{\psi} H_{\perp}\right|_{S}=-\left.£_{H_{\perp}} \psi\right|_{S}=0 .
$$

The conditions (45) and (46) are consistent with the ones in [6, 9, 10, 11] for dynamical trapping horizons. These conditions then imply that

$$
\left.£_{\xi} \xi\right|_{S}=0,\left.\quad £_{\xi} \varrho\right|_{S}=0,\left.\quad £_{\xi} j\right|_{S}=0,
$$

the stationary untrapped boundary conditions (I,II,III) are satisfied.

By equation (43), the Hamiltonian associated with the evolution vector (44) is then given by

$$
\left.\mathbb{E}(\xi)=\oint_{S}\left(f(\varrho, j)-h(\varrho, j) H^{2}\right)\right) \varrho .
$$

By requiring that the energy expression gives the standard value for Kerr black holes at marginal surface $H=0$,

$$
\mathbb{E}_{\text {horizon }}=\frac{\sqrt{\mathbb{R}^{4}+4 \mathbb{J}^{2}}}{2 \mathbb{R}}
$$

we obtain

$$
f(\varrho, j)=\frac{\sqrt{\mathbb{R}^{4}+4 \mathbb{J}^{2}}}{8 \pi \mathbb{R}^{3}} .
$$

The other free function $h(\varrho, j)$ can be chosen such that the energy is proportional to the Hawking energy and gives ADM mass at spatial infinity, this implies that

$$
h(\varrho, j)=\frac{\sqrt{\mathbb{R}^{4}+4 \mathbb{J}^{2}}}{32 \pi \mathbb{R}} .
$$

Then the Hamiltonian associated with $\xi$,

$$
\mathbb{E}(\xi)=\frac{\sqrt{\mathbb{R}^{4}+4 \rrbracket^{2}}}{2 \mathbb{R}}\left(1-\frac{1}{16 \pi} \oint_{S} H^{2} \varrho\right)
$$

provides a suitable choice for the energy expression.

Note that the vector on $S$,

$$
\left.\xi\right|_{S}=\frac{\sqrt{\mathbb{R}^{4}+4 \rrbracket^{2}}}{32 \pi \mathbb{R}} H_{\perp}-\Omega \psi
$$

reduces to the Kodama vector

$$
\xi_{\text {Kodama }}=\frac{\mathbb{R}}{32 \pi} H_{\perp},
$$

in spherically symmetric spacetimes [25]. Thus equation (53) is a generalized Kodama vector for non-spherically symmetric spacetimes. 


\section{Discussion}

In summary, for quasi-local regions bounded by an untrapped or marginal 2-surface, a functional differentiable Hamiltonian can be defined with the boundary conditions which fix the evolution vector (equation (25)), the area element (equation (26)) and the angular momentum surface density (equation (41)) on the 2-surface. As a consequence, a preferred expression of quasi-local energy for these "stationary untrapped boundary conditions" is given, which allows the geometry outside to be dynamical and to admit gravitational and other radiation. The quasi-local energy expression (equation (52)) generalizes the Hawking energy to include angular momentum. On the boundary, the evolution vector associated with this expression is a generalization of the Kodama vector (equation (53)). These results generalize the conserved quantities for Isolated Horizons so as to provide covariant conserved quantities for general untrapped regions.

In equation (31), the evolution vector on the boundary is chosen to be proportional to the dual expansion vector. Alternatively, we can use the unit dual expansion vector. If $H$ is not null $(|H| \neq 0)$, there is a set of uniquely determined unit normal vectors for the 2-surface, given by

$$
\hat{e}_{0}=\frac{H_{\perp}}{|H|} \quad \hat{e}_{1}=\frac{H}{|H|},
$$

where $|H|=\sqrt{H \cdot H}=\sqrt{k\left(e_{1}\right)^{2}-k\left(e_{0}\right)^{2}}$. If we choose the evolution vector $\xi$ such that $\left.\xi\right|_{S}=\hat{e}_{0}$, then by equation (30), this leads to the energy expression

$$
\left.\mathbb{E}(\xi)=\oint_{S}\left(f(\varrho)-\sqrt{k\left(e_{1}\right)^{2}-k\left(e_{0}\right)^{2}}\right)\right) \varrho .
$$

Here we have only one free function $f(\varrho)$. A natural requirement is that the expression should give ADM energy at spatial infinity. Assuming that we can embed the 2-surface isometrically into Minkowski spacetime, let $k_{0}\left(e_{1}\right)$ be the trace of extrinsic curvature with respect to $e_{1}$, for the 2 -surface in Minkowski spacetime, then the choice $f(\varrho)=k_{0}\left(e_{1}\right)$ gives the Kijowski-Liu-Yau quasi-local energy [26, 27]. In the special $k\left(e_{0}\right)=0$ slice, the

Kijowski-Liu-Yau energy equals the Brown-York energy [28, 29]. There are nice positive energy theorems for these expressions [27, 30]. However, $\hat{e}_{0}$ fails to be defined in the null case. Moreover, the requirement of isometric embedding assumes that the full induced 2-metric is fixed (rather than just the area element). Thus it seems that this choice is not suitable for the cases involving dynamical black holes.

The stationary untrapped boundary conditions can be compared with the Isolated Horizon boundary conditions. The untrapped surface $S$ together with the associated evolution vector on the boundary $\left.\xi\right|_{S}$ constitute a timelike hypersurface. In the limit when the dual expansion vector $H_{\perp}$ is null, $S$ reduces to a marginal surface, the hypersurface reduces to a non-expanding horizon (null 3-surfaces in spacetime on which the expansion with respect to any null normal is zero) [12. This suggests a generalization of the non-expanding horizons to "stationary untrapped hypersurfaces" (with the boundary conditions I, II, III) describing the equilibrium states of untrapped 
surfaces with conservation of the area (with area radius $\mathbb{R}$ ), the energy $\mathbb{E}$, and the angular momentum $\mathbb{J}$.

\section{Acknowledgments}

The author thanks Sean A. Hayward, James M. Nester and Laszlo B. Szabados for helpful discussions. This work was supported by the National Natural Science Foundation of China under grant numbers 10375081, 10375087 and 10771140, by Shanghai Pujiang Talent Program, by Shanghai Education Development Foundation Shuguang Award and by NCTS Taiwan.

\section{References}

[1] S. A. Hayward, "General laws of black-hole dynamics", Phys. Rev. D 49 (1994) 6467.

[2] A. Ashtekar, C. Beetle, O. Dreyer, S. Fairhurst, B. Krishnan, J. Lewandowski and J. Wisniewski, "Generic isolated horizons and their applications", Phys. Rev. Lett. 85 (2000) 3564.

[3] A. Ashtekar, C. Beetle and J. Lewandowski, "Mechanics of rotating isolated horizons", Phys. Rev. D 64 (2001) 044016.

[4] A. Ashtekar, C. Beetle and J. Lewandowski, "Geometry of generic isolated horizons", Class. Quantum Grav. 19 (2002) 1195.

[5] A. Ashtekar and B. Krishnan, "Dynamical horizons: energy, angular momentum, fluxes, and balance laws", Phys. Rev. Lett. 89 (2002) 261101.

[6] A. Ashtekar and B. Krishnan, "Dynamical horizons and their properties", Phys. Rev. D 68 (2003) 104030 .

[7] S. A. Hayward, "Energy and entropy conservation for dynamical black holes", Phys. Rev. D 70 (2004) 104027.

[8] I. Booth and S. Fairhurst, "The first law for slowly evolving horizons", Phys. Rev. Lett. 92 (2004) 011102.

[9] E. Gourgoulhon, "Generalized Damour-Navier-Stokes equation applied to trapping horizons", Phys. Rev. D 72 (2005) 104007.

[10] I. Booth and S. Fairhurst, "Horizon energy and angular momentum from a Hamiltonian perspective", Class. Quantum Grav. 22 (2005) 4515.

[11] S. A. Hayward, "Angular momentum conservation for dynamical black holes", Phys. Rev. D 74 (2006) 104013.

[12] A. Ashtekar and B. Krishnan, "Isolated and dynamical horizons and their applications", Living Review in Relativity 7 (2004) 10.

[13] L. B. Szabados, "Quasi-local energy-momentum and angular momentum in GR: a review article", Living Review in Relativity 7 (2004) 4.

[14] S. C. Anco and R. S. Tung, "Covariant Hamiltonian boundary conditions in general relativity for spatially bounded space-time regions", J. Math. Phys. 43 (2002) 5531; erratum 45 (2004) 2109.

[15] S. C. Anco and R. S. Tung, "Properties of the symplectic structure of general relativity for spatially bounded space-time regions", J. Math. Phys. 43 (2002) 3984; erratum 45 (2004) 2108.

[16] T. Regge and C. Teitelboim, "Role of surface integrals in the Hamiltonian formulation of general relativity", Annals of Physics 88 (1974) 286.

[17] L. B. Szabados, "On a class of 2-surface observables in general relativity", Class. Quantum Grav. 23 (2006) 2291.

[18] L. B. Szabados, "Two-dimensional Sen connections in general relativity", Class. Quantum Grav. 11 (1994) 1833. 
[19] J. Lee and R. M. Wald, "Local symmetries and constraints", J. Math. Phys. 31 (1990) 725.

[20] J. M. Nester, "A covariant Hamiltonian for gravity theories", Mod. Phys. Lett. A 6 (1991) 2655; J. M. Nester, "Some progress in classical canonical gravity", in Directions in General Relativity, ed. B. L. Hu, M. P. Ryan and C. V. Vishveshwara (Cambridge University Press) Vol I (1993) 245.

[21] R. M. Wald, "Black hole entropy is Noether charge", Phys. Rev. D 48 (1993) 3427.

[22] R. M. Wald and A. Zoupas, "General definition of "conserved quantities" in general relativity and other theories of gravity", Phys. Rev. D 61 (2000) 084027.

[23] C. M. Chen, J. M. Nester and R. S. Tung, "Hamiltonian boundary term and quasilocal energy flux", Phys. Rev. D 72 (2005) 104020.

[24] S. W. Hawking, "Gravitational radiation in an expanding universe", J. Math. Phys. 9 (1968) 598.

[25] H. Kodama, "Conserved energy flux from the spherically symmetric system and the back reaction problem in the black hole evaporation", Prog. Theor. Phys. 63 (1980) 1217.

[26] J. Kijowski, "A simple derivation of canonical structure and quasi-local Hamiltonians in general relativity", Gen. Rel. Grav. 29 (1997) 307.

[27] C.-C. M. Liu and S.-T. Yau, "Positivity of quasilocal mass", Phys. Rev. Lett. 90 (2003) 231102.

[28] J. D. Brown, J. W. York, Jr., "Quasilocal energy and conserved charges derived from the gravitational action", Phys. Rev. D 47 (1993) 1407.

[29] J. D. Brown, S. R. Lau and J. W. York, Jr., "Action and energy of the gravitational field", Ann. Phys. (N.Y.) 297 (2002) 175.

[30] Y. Shi and L.-F. Tam, "Positive mass theorem and the boundary behaviors of compact manifolds with nonnegative scalar curvature", J. Differ. Geom. 62 (2002) 79. 